\title{
Fast Reactions Involving Radical-Cations During their Geminate Recombination as Studied by the OD ESR Method
}

\author{
V. O. Saik, O. A. Anisimov, V. V. Lozovoy, and Yu. N. Molin \\ Institute of Chemical Kinetics and Combustion, Novosibirsk 630090, USSR, and Novosibirsk \\ State University, Novosibirsk 630090, USSR
}

Z. Naturforsch. 40 a, 239-245 (1985); received November 22, 1984

\begin{abstract}
Dedicated to Professor Schulte-Frohlinde on the Occasion of his $60^{\text {th }}$ Anniversary
The new method of optically detected ESR (OD ESR) of radical-ion pairs has been used to study fast radical-cation reactions in non-polar liquid solutions. Dimeric radical-cations have been observed to occur within the time of geminate recombination with radical-ions; their generation rate has been determined. The spectral line broadening with increasing acceptor concentration has been associated with ion-molecular charge transfer; the rate of this process has also been determined.
\end{abstract}

It is well-known that radiolysis of hydrocarbons with positive charge acceptors is accompanied by generation of radical-cations. Radical-cations in frozen matrices were investigated by a standard ESR technique. Also ESR spectra have been taken [1-3] from dimeric radical-cations of aromatic hydrocarbons and of methyl derivated aromatic hydrocarbons and some conclusions on the structure of dimeric radical-cations have been drawn. The highly sensitive method of optically detected ESR (OD ESR) [4] has allowed for analogous investigations of short-lived (about $10^{-7} \mathrm{~s}$ ) radical-cations involved in the primary track recombination in liquid solutions [5]. In the present work, the OD ESR method is employed to study fast radical-cation reactions in non-polar liquid solutions including charge transfer and dimerization. So far radicalcation reactions have been investigated by optical methods combined with pulse radiolysis [6]. Such investigations, however, are faced with the problem of identifying the optical absorption spectra of radical-cations and of their often overlapping products. Moreover, optical methods are inapplicable to degenerate processes, e.g., reactions of ion-molecular charge transfer.

Reprint requests to Dr. V. O. Saik, Institute of Chemical Kinetics and Combustion, Novosibirsk-90, USSR.

\section{Experimental Technique}

The ESR spectra of radical-cations were investigated on the setup for optical detection of ESR described in detail earlier [7]. Radical-ion pairs arose under continuous X-ray irradiation (dose rate of $85 \mathrm{krad} / \mathrm{h}$ ) directly in the cavity of a VARIAN-E-3 ESR spectrometer. The OD ESR signals were detected by falling luminescence of a sample and recorded as the first derivatives by the phase-sensitive detection technique.

The samples were cooled with a nitrogen gas flow from a dewar vessel; the temperature was monitored with a thermocouple soldered inside a quartz capillary tube placed in the solution. The temperature was maintained constant to $\pm 1{ }^{\circ} \mathrm{C}$.

Trans-decalin and squalane $(2,6,10,15,19,23$-hexamethyltetracosane) were purified by passing through a column with activated silica gel; the solvent purities were checked by the ultraviolet transmission cutoff which was $200 \mathrm{~nm}$ and $227 \mathrm{~nm}$ for transdecalin and squalane, respectively. (The transmission cut-off corresponds to the optical density of a 1-cm-layer equal to unity.) The solutions were degassed by repeated freeze-pump-thaw cycles.

Durene, hexamethylbenzene (HMB), p-terphenyl$\mathrm{d}_{14}$, naphthalene and biphenyl were purified by recrystallization. Tetramethylethylene (TME) (FLUKA, 99.5\%) were used without additional purification. Triethylamine (TEA) was distilled.

0340-4811 / 85 / 0300-0239\$01.30/0. - Please order a reprint rather than making your own copy. 
The recombination luminescence kinetics were taken on a setup described earlier [8-9].

\section{Results}

Figures 1, 2 show the central parts of OD ESR spectra for solutions of p-terphenyl- $\mathrm{d}_{14}$ and a hole acceptor (either TME or durene) at various concentrations in trans-decalin and squalane, respectively. The central spectral line belongs to radical-ions of p-terphenyl- $\mathrm{d}_{14}$ whose spectrum is well-known [4]; the rest of the lines corresponds to TME and durene radical-cations. At a low TME concentration (Fig. la) the splitting observed corresponds to a monomeric $\mathrm{TME}^{+}$radical-cation $[9,10]$ resulting from a solvent hole capture by a TME molecule. As the TME concentration increases, there arise new spectral lines with approximately twice as low splitting (see Table 1) pertaining to dimeric $\mathrm{TME}_{2}^{+}$radical-cations [10]. The intensity of these lines increases with concentration. What is more, a rise in the TME concentration broadens the line widths and shifts the components towards the centre. In the case of high acceptor concentrations, when the spectrum shows only dimeric cation signals, a rise in the concen-

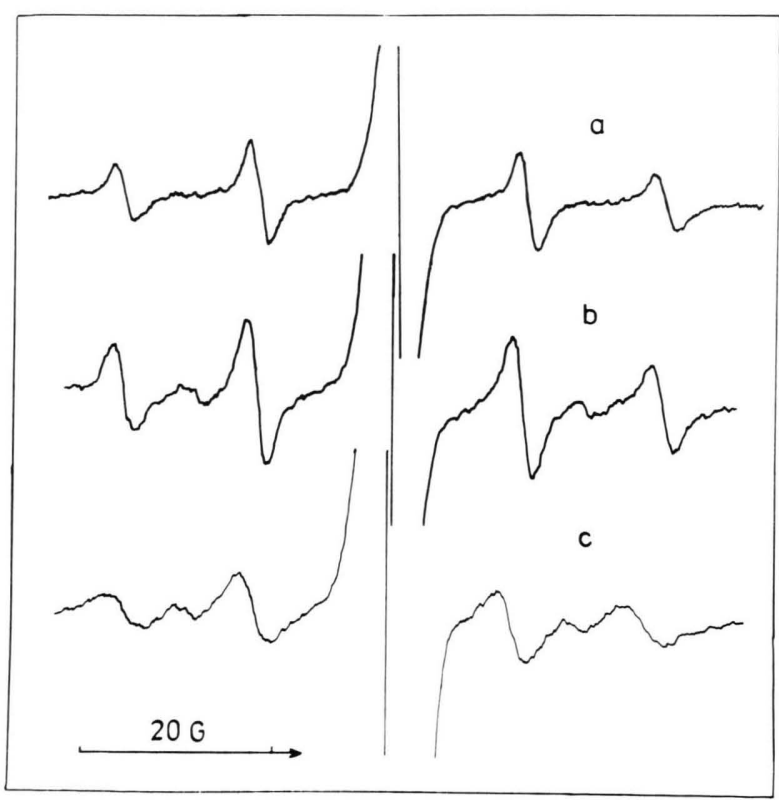

Fig. 1. OD ESR spectra (central part) for pairs of TME radical-cations and p-terphenyl- $\mathrm{d}_{14}$ radical-anions in transdecalin at various TME concentrations: (a) $3 \cdot 10^{-3} \mathrm{M}$, (b) $9 \cdot 10^{-3} \mathrm{M}$, (c) $1.8 \cdot 10^{-2} \mathrm{M}$; p-terphenyl-d $\mathrm{d}_{14}$ concentration is $3.7 \cdot 10^{-4} \mathrm{M}$ everywhere, temperature is $299 \mathrm{~K}$.

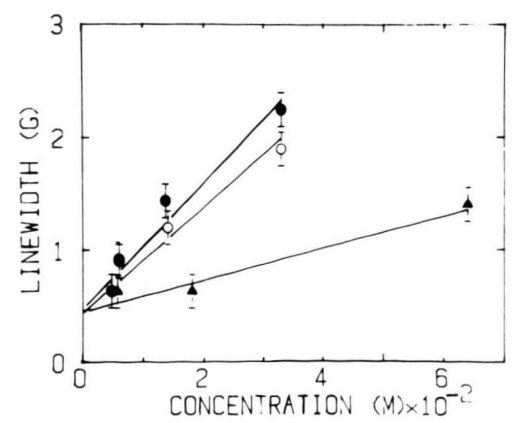

Fig. 3. Plots of individual OD ESR line widths for monomeric radical-cations of $\operatorname{HMB}(\mathbf{\Delta})$, durene $(0)$ and dimeric radical-cations of durene $(\bullet)$ vs concentrations of $\mathrm{HMB}$ and durene, respectively, in squalane. Temperature is $297 \mathrm{~K}$ radical-cations and $\mathrm{p}$-terphenyl- $\mathrm{d}_{14}$ radical-anions in squa lane at various durene concentrations: (a) $10^{-2} \mathrm{M}$, (b) 2 $10^{-2} \mathrm{M}$, (c) $10^{-1} \mathrm{M}$; p-terphenyl- $\mathrm{d}_{14}$ concentration is 4.5 $10^{-4} \mathrm{M}$ everywhere, temperature is $299 \mathrm{~K}$. 
tration also broadens the hfs lines and shifts the spectral components to the centre. In the case of durene radical-cations, the spectra vary in the same manner (Figure 2). The concentration dependences of the line widths for radical-cations are shown in Fig. 3 for some systems studied. Figure 4 depicts OD-ESR spectra for HMB radical-cations in squalane at different temperatures. As the temperature falls off, the signals from dimeric cations first reduce, then vanish. Figure 5 presents OD ESR spectra from TME radical-cations in squalane at various temperatures. A fall in temperature leads to a decrease in the line width. In the case of low acceptor concentrations, a fall of temperature does not affect the hfs line widths within the same temperature range. Figure 6 shows the central part of the OD ESR spectrum from durene radical-cations for various oxygen concentrations in the solution. Oxygen in the solution is seen to reduce the signals from dimeric cations compared to those from monomeric ones.

\section{Discussion}

The radiolysis process in non-polar solutions can be described by the simplified scheme [4]

$$
\begin{aligned}
& \mathrm{S} \rightarrow \mathrm{S}^{+}+\mathrm{e}^{-}, \\
& \mathrm{e}^{-}+\mathrm{A} \rightarrow \mathrm{A}^{-}, \\
& \mathrm{S}^{+}+\mathrm{D} \rightarrow \mathrm{D}^{+}+\mathrm{S}, \\
& \mathrm{D}^{+}+\mathrm{A}^{-} \rightarrow \mathrm{D}+\mathrm{A}^{*}, \\
& \mathrm{~A}^{*} \rightarrow \mathrm{A}, \\
& \mathrm{D}^{+}+\mathrm{D} \rightarrow \mathrm{D}_{2}^{+}, \\
& \mathrm{D}_{2}^{+}+\mathrm{A}^{-} \rightarrow 2 \mathrm{D}+\mathrm{A}^{*} .
\end{aligned}
$$

Here $\mathrm{S}$ is a solvent molecule; $\mathrm{A}, \mathrm{D}$ are electron and hole acceptors, respectively. The OD ESR spectrum arises due changes in the spin state of $\mathrm{D}^{+} / \mathrm{A}^{+}[4]$ and $\mathrm{D}_{2}^{+} / \mathrm{A}^{-}$radical-ion pairs. As mentioned above, the fact that a rise in the whole acceptor concentration results in spectral lines with half as low hfs constants can be explained by the formation of dimeric $\mathrm{D}_{2}^{+}$radical-cations. The spin density of an unpaired electron in the dimeric cations proves to be shared between two acceptor molecules, so that the hfs constants should reduce by half. Actually, the hfs constants of dimeric cations are less than half those of monomeric cations. This discrepancy has already been discussed by Howarth and Fraenkel [2] who believe this to stem from a change of the McConnel

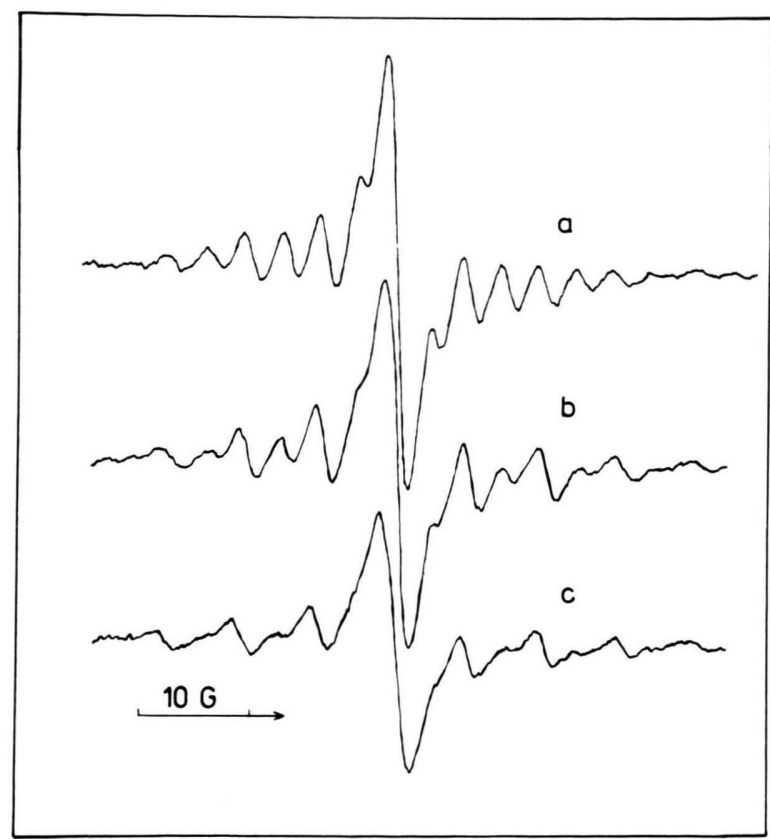

Fig. 4. OD ESR spectra (central part) for pairs of HMB radical-cations and p-terphenyl- $\mathrm{d}_{14}$ radical anions in squalane with concentrations of $\mathrm{HMB} 3 \cdot 10^{-2} \mathrm{M}$ and of p-terphenyl- $\mathrm{d}_{14}$ of $10^{-3} \mathrm{M}$ at temperatures: (a) $299 \mathrm{~K}$, (b) $280 \mathrm{~K}$, (c) $271 \mathrm{~K}$.

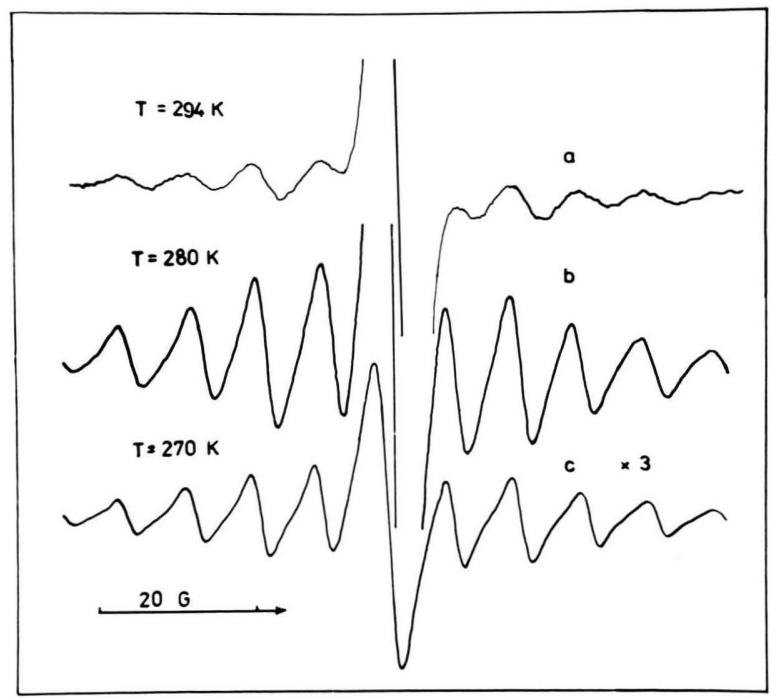

Fig. 5. OD ESR spectra (central part) for pairs of TME radical-cations with $\mathrm{p}$-terphenyl- $\mathrm{d}_{14}$ radical-anions in squalane at various temperatures. Concentrations of TME $8.5 \cdot 10^{-2} \mathrm{M}$, of p-terphenyl- $\mathrm{d}_{14} 10^{-3} \mathrm{M}$. 
factor $Q$ in the relation $a_{\mathrm{hfi}}=Q \varrho$ depending on the electron charge on the neighbouring carbon atom.

In pulse radiolysis experiments with radicalcations detected by optical absorption [6], dimeric radical-cations were assumed to arise by reaction (6). A decrease in the fraction of dimeric radicalcations in the OD ESR spectrum observed at lowering temperature (see Fig. 4) can be readily interpreted by reaction (6). Indeed a rise in the solution viscosity with falling temperature hampers collisions of monomeric radical-cations with neutral molecules and thus reduces the fraction of $\mathrm{D}_{2}^{+}$ arising during the ESR spectrum formation (as a rule, this time period is limited by the spin-lattice relaxation time of radical-ions). Also, the generation of dimeric radical-cations by reaction (6) is confirmed by experiments with amounts of oxygen (Figure 6). A collision of a radical-ion partners with oxygen paramagnetic molecules leads to spin correlation losses of the pair. Such a pair cannot contribute to the ODESR spectrum. The longer the lifetime of this pair, the higher the probability of its collision with an oxygen molecule. The lifetime of pairs involving monomeric radical-cations is shortened by reaction (6). As a result, the effect of oxygen on monomeric lines is smaller than that on dimeric ones.

So experiment demonstrates that in the geminate recombination of TME, HMB and durene radicalcations in saturated hydrocarbons, their collisions with neutral molecules result in dimeric radicalcations, the relative spin orientation in the newly formed $D_{2}^{+} / A^{-}$pairs being the same as that in $\mathrm{D}^{+} / \mathrm{A}^{-}$ones.

As mentioned above, an increase in the acceptor concentration leads to a broadening of the hfs components and shifts them towards the centre for monomeric and dimeric radical-cations. These changes point to frequency migration in the ESR spectrum. The frequency migration results perhaps from the process of ion-molecular charge transfer,

$$
\mathrm{A}^{+}+\mathrm{A} \rightarrow \mathrm{A}+\mathrm{A}^{+},
$$

in a spectrum of monomeric radical-cations, and from the reaction of a molecule exchange,

$$
\mathrm{AA}^{+}+\mathrm{A}^{\prime} \rightarrow \mathrm{AA}^{\prime+}+\mathrm{A},
$$

in a spectrum of dimeric radical-cations. Both processes depend on the solvent viscosity and deceler-

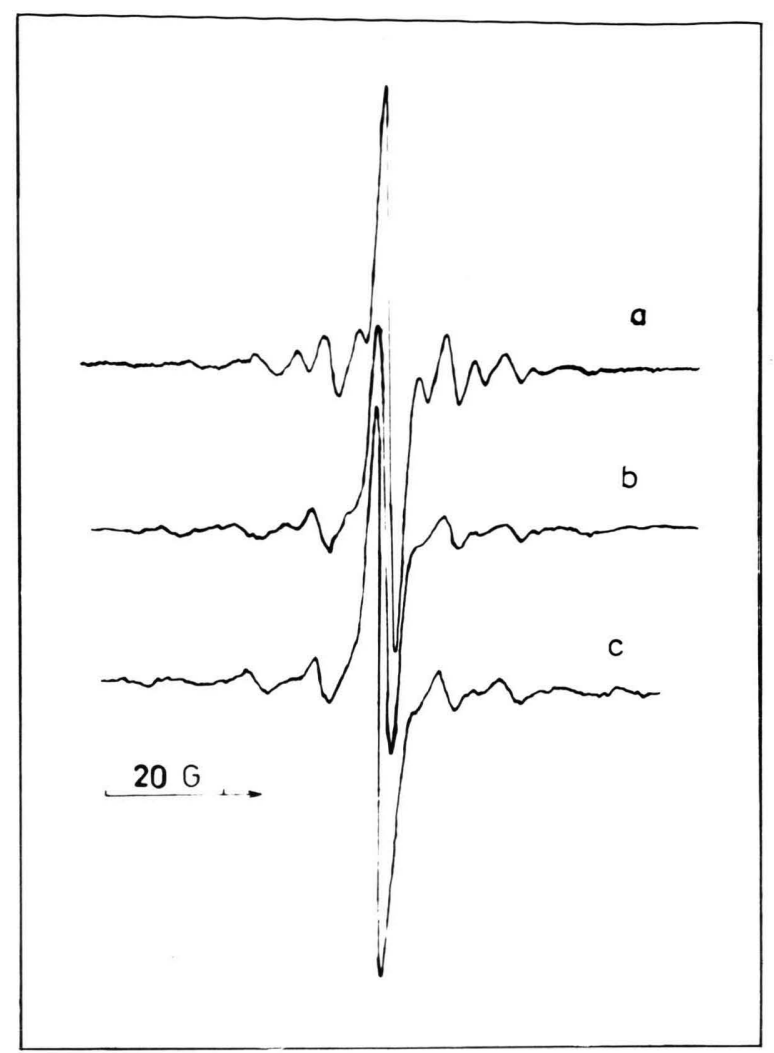

Fig. 6. OD ESR spectra for pairs of durene radical-cations with p-terphenyl- $\mathrm{d}_{14}$ radical-anions in squalane at concentrations of durene $4.7 \cdot 10^{-3} \mathrm{M}$ and of p-terphenyl- $\mathrm{d}_{14}$ $4.4 \cdot 10^{-4} \mathrm{M}$ with amounts of oxygen in the solution. Oxygen pressure above the sample is (a) about $2 \cdot 10^{-4}$ torr, (b) about 35 torr, (c) about 70 torr. Temperature is $299 \mathrm{~K}$. Spectra (b) and (c) are multiplied by the factor 1.2 and 2.5 respectively.

ate with falling temperature, which leads to narrowing of the hfs components (Figure 5). The concentration dependences of the width and shift of the hfs components allow the rate constants of reactions (8) and (9) to be estimated. The rate constants were computed by simulating the spectra. In the general case, a precise analysis of OD ESR spectral variations due to reactions (8), (9) is quite a problem. However, in the conditions of our experiments, the shape of the OD ESR spectrum is fairly well described in terms of the Zeeman transitions in crossing constant and microwave magnetic fields independently for radical-cation and -anion spins [11, 12], which case is analogous to that of normal ESR. Therefore, it proves to be possible to employ sta- 


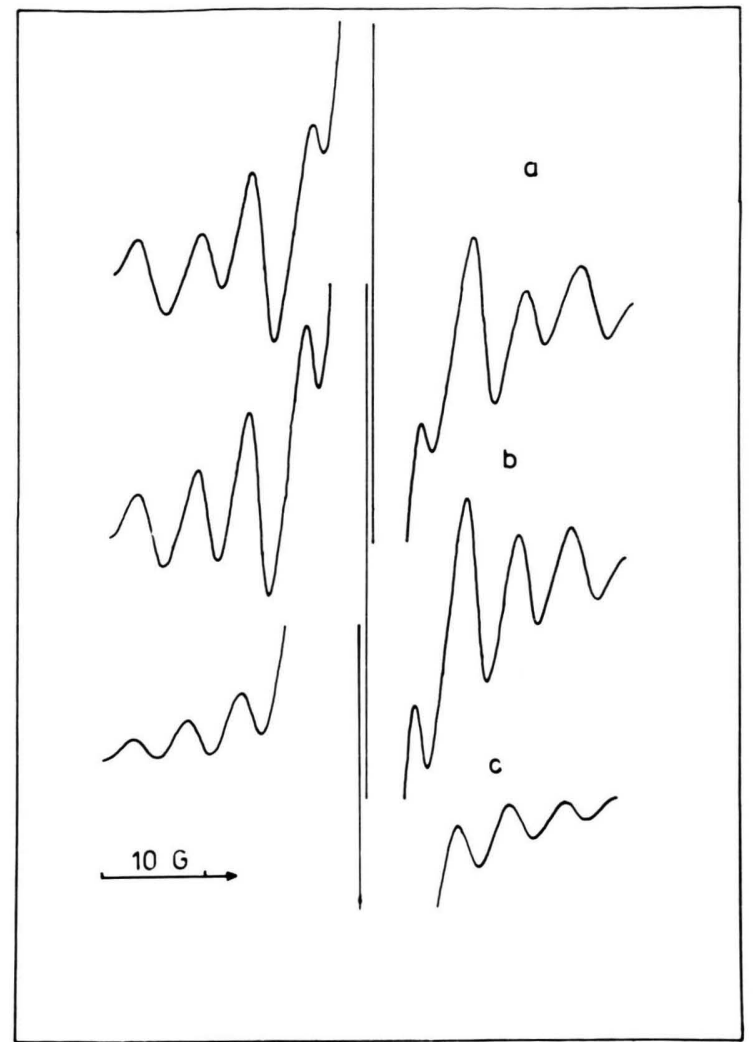

Fig. 7. Spectra from Fig. 2 simulated for the rate constants from Table 1.

tionary solutions of the McConnel equations [13] to simulate OD ESR spectra. The so obtained reaction rate constants are listed in Table 1. Figure 7 shows some ODESR spectra simulated with parameters from Table 1.

ODESR signals from recombining pairs have been shown [14] to be saturated essentially within the experimental range of $H_{1}$ fields from 0 to $0.75 \mathrm{G}$. The OD ESR signal intensity has been calculated [14] as a function of microwave power taking into account the true lifetime distribution of recombining pairs, which is known [15] to obey the function $F(t) \infty t^{-3 / 2}$. The line shape under saturation has been shown to depend on the three parameters $T_{1}^{\prime}$, $T_{2}$ and $T_{\mathrm{e}}$, where $T_{2}$ is a radical spin-spin relaxation time, $T_{\mathrm{e}}$ a frequency migration time and $T_{1}^{\prime}$ a time of spin correlation losses in a recombining pair. Other factors absent, spin correlation losses are determined by the time $T_{1}$ of spin-lattice relaxation of two partners, $T_{1}^{\prime}=T_{1}$. In the case of reaction (6),

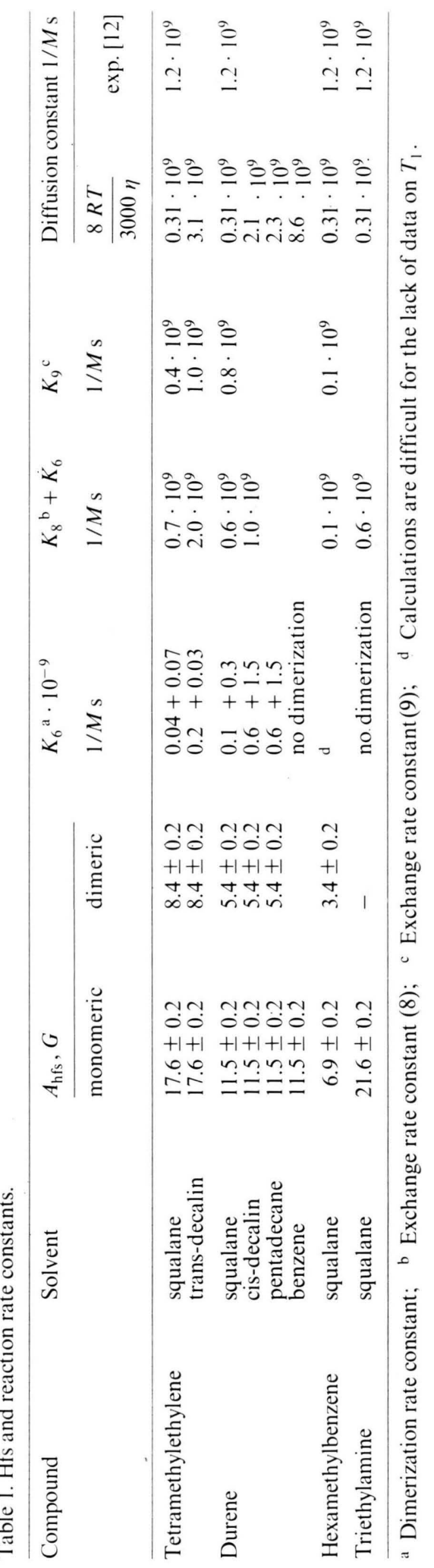




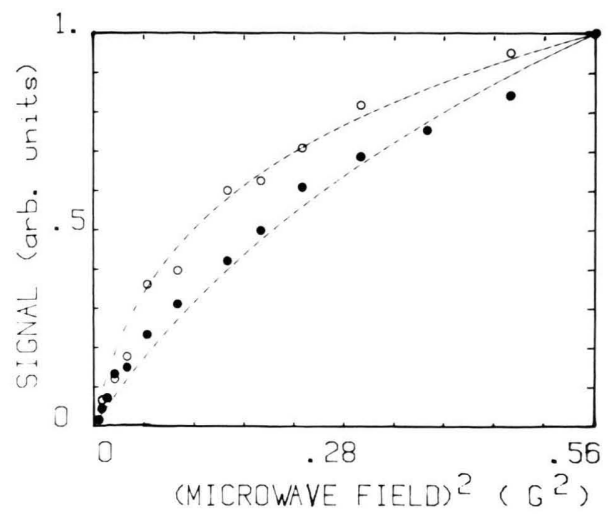

Fig. 8. Plots of individual line intensities vs $H_{1}$ for durene radical-cations in solutions of: $3 \cdot 10^{-3} \mathrm{M}$ durene and $5 \cdot 10^{-4} \mathrm{M}$ p-terphenyl- $\mathrm{d}_{14}$ in squalane $(0) ; 1.2 \cdot 10^{-2} \mathrm{M}$ durene and $5 \cdot 10^{-4} \mathrm{M}$ p-terphenyl- $\mathrm{d}_{14}$ in squalane $(\bullet)$. Dashed lines calculated as described in [14], top curve: $T_{1}^{\prime}=2000 \mathrm{~ns}, T_{2}=250 \mathrm{~ns}, T_{\mathrm{e}}=560 \mathrm{~ns}$; bottom curve: $T_{1}^{\prime}=$ $400 \mathrm{~ns}, 1 / T_{2}=0, T_{\mathrm{e}}=140 \mathrm{~ns}$.

$T_{1}$ for monomeric radical-cations obeys the expression

$$
1 / T_{1}^{\prime}=1 / T_{1}+1 / \tau
$$

where $\tau$ is the time of reaction (6). This relation can be employed to determine the generation rate of dimeric radical-cations. The time $T_{1}$ can be estimated by decay of a time-resolved magnetic effect detectable by the method described by Brocklehurst et al. [16]. Experiment demonstrates $T_{1}$ to exceed $2 \mu \mathrm{s}$ in radical-ion pairs involving durene and TME radicalcations. In the case of pairs with HMB radicalcations, $T_{1}$ cannot be estimated since the recombination luminescence kinetics for solutions with $\mathrm{HMB}$ have a long-lived component which hinders the observation of magnetic effects.

Figure 8 depicts the saturation curves for the first high field's hfs component of a durene radicalcation in squalane at various durene concentrations. For a durene concentration of $1.2 \cdot 10^{-2} \mathrm{M}$ the line width is $1 \mathrm{G}$, which is noticeably wider than that in dilute solutions (about $0.3-0.4 \mathrm{G}$ ) with negligible frequency migration. In this case, the time $T_{2}$ may be set to be determined mainly by the charge transfer time $T_{\mathrm{e}}$. Hence, neglecting the intrinsic time $T_{2}$ of a radical and having determined $T_{\mathrm{e}}$ from the line width, one can select $T_{1}^{\prime}$ so that the calculated saturation curve may be in best agreement with experimental points. Within the concentration range studied, $T_{1}^{\prime}$ is $400 \mathrm{~ns}$. Since $T_{1}$ exceeds $2 \mu \mathrm{s},[10]$ affords $\tau$ to equal $400-500 \mathrm{~ns}$.

As seen from Fig. 8, a fourfold decrease of durene concentration leads to a change in the saturation curve shape. This should be expected since both $\tau$ and $T_{\mathrm{e}}$ are inversely proportional to concentration and increase by 4 times. The curve calculated for corresponding $\tau$ and $T_{\mathrm{e}}$ values fairly well agrees with experiment provided the intrinsic radical relaxation time $T_{2}$ is set equal to 250-400 ns. (In contrast to the previous case, the intrinsic relaxation time cannot be neglected because of the small line width at a concentration of $3 \cdot 10^{-3} \mathrm{M}$.) The so selected $T_{2}$ does not contradict the line width observed in dilute solutions.

Thus, at sufficiently high acceptor concentrations, saturation curves can be used to estimate the time $\tau$ and the constant $k_{6}$ (see Table 1).

As demonstrated by experiment, TEA solutions contain only monomeric radical-cations, which fact points to the absence of reaction (6). Also it is interesting to note that in the case of TME and durene in aromatic solvents (benzene, p-xylene) no cation dimerization occurs.

\section{Conclusion}

The systems studied have demonstrated the possibility of applying the OD ESR method to fast reaction studies. The examples considered have vividly shown potentialities and limitations of the OD ESR method. For instance, it is no problem to reliably determine the ion-molecular charge transfer constants from the spectral line broadening, while dimerization constants can be evaluated only indirectly. More reliable information on these constants could be supplied by a time-resolved version of the OD ESR method [15].

In conclusion, the authors would like to thank Prof. R. M. Mehnert who kindly supplied us with unpublished results and kinetic data on some shortlived radicals, Dr. V. I. Melikhov for assistance in experiments and useful discussions, and Drs. S. V. Anishik and S. N. Smirnov for fruitful discussions. 
[1] I. C. Lewis and L. S. Singet, J. Chem. Phys. 43, .2712 (1965).

[2] O. W. Hewath and G. K. Fraenkel. J. Chem. Phys. 52, $6258(1970)$.

[3] C. Thowson and W. J. Macculloch, Mol. Phys. 19, 817 (1970).

[4] Yu. N. Molin and O. A. Anisimov, Radiat. Phys. Chem. 21, 77 (1983).

[5] V. M. Grigoryants, O. A. Anisimov, and Yu. N. Molin, Zh. Strukt. Khim. 23, 4 (1982).

[6] R. Mehnert, O. Brede, and Gy. Cserep, Radiochem. Radional. Lett. 47 [3], 173 (1981).

[7] O. A. Anisimov, V. M. Grigoryants, V. I. Korsunsky, V. I. Melekhov, and Yu. N. Molin, DAN SSSR 260, 1151 (1981).

[8] O. A. Anisimov, V. L. Bizyaev, N. N. Lukzen, V. M. Grigoryants, and Yu. N. Molin, DAN SSSR 272, 383 (1983).

[9] O. A. Anisimov, V. L. Bizyaev, N. N. Lukzen, V. M. Grigoryants, and Yu. N. Molin, Chem. Phys. Lett. 101, 131 (1983).
[10] S. Ya. Pshezhetsky, A. G. Kotov, V. K. Milinchuk, V. A. Roginsky, and V. I. Tupikov, ESR of Free Radicals in Radiation Chemistry, Khimiya, Moscow 1972, p. 267.

[11] A. B. Doktorov, O. A. Anisimov, A. I. Burshtein, and Yu. N. Molin, Chem. Phys. 71, 1 (1982).

[12] V. O. Saik, N. N. Lukzen, V. M. Grigoryants, O. A. Anisimov, A. B. Doktorov, and Yu. N. Molin, Chem. Phys. 84, 421 (1984).

[13] K. I. Zamaraev, Yu. N. Molin, and K. M. Salikhov, Spin Exchange, Nauka, Novosibirsk 1977.

[14] N. N. Lukzen, V. O. Saik, O. A. Anisimov, and Yu. N. Molin (in press).

[15] J. P. Smith and A. D. Trifunac, J. Phys. Chem. 85, 1645 (1981).

[16] B. Brocklehurst, R. S. Dixon, E. M. Gardy, V. J. Lopata, M. J. Quinn, A. Singh, and F. P. Sargent, Chem. Phys. Lett. 28, 361 (1974). 